\title{
CASP-1, -2 and -5 gene polymorphisms and cancer risk: A review and meta-analysis
}

\author{
YING-LI PAN ${ }^{1}$, WEI LIU ${ }^{2}$, CAI-XIANG GAO ${ }^{1}$, ZUO SHANG $^{1}$, LI-JUAN NING ${ }^{3}$ and XING LIU² \\ Departments of ${ }^{1}$ Nursing, ${ }^{2}$ General Surgery and ${ }^{3}$ Cardiology, The Fourth Affiliated Hospital of China Medical University, \\ Shenyang, Liaoning 110032, P.R. China
}

Received November 20, 2012; Accepted January 9, 2013

DOI: $10.3892 /$ br.2013.98

\begin{abstract}
Accumulating evidence suggests the CASP gene family is important in the development of carcinogenesis. These genetic polymorphisms have been extensively investigated as a potential risk factor for cancer, but results have been inconclusive. This Human Genome Epidemiology (HuGE) review and meta-analysis was performed to investigate the associations between CASP-1, -2 and -5 and cancer risk. A literature search of Pubmed, Embase, Web of Science and CBM databases was conducted from inception through September 1st, 2012. Four case-control studies with a total of 1,592 cancer cases and 1,833 healthy controls were included in the present meta-analysis. Crude odds ratios (ORs) with $95 \%$ confidence intervals (CIs) were used to assess the strength of association. Five polymorphisms were examined, including rs501192 (G>A), rs4647297 (C>G), rs507879 (T>C), rs3181320 $(\mathrm{G}>\mathrm{C})$ and rs523104 $(\mathrm{G}>\mathrm{C})$. Meta-analysis results showed that the $\mathrm{rs} 3181320^{*} \mathrm{C}$ allele/carrier were associated with increased risk of various types of cancers $(\mathrm{OR}=1.26$; 95\% CI, 1.04-1.54; $\mathrm{P}=0.020$ and $\mathrm{OR}=1.33$; 95\% CI, 1.00-1.75; $\mathrm{P}=0.047$, respectively). However, similar associations were not found in the rs501192, rs4647297, rs507879 and rs523104 polymorphisms (all $\mathrm{P}>0.05$ ). Results from the current meta-analysis suggest that the $\mathrm{rs} 3181320^{*} \mathrm{C}$ allele/carrier in CASP-5 gene are potential risk factors for cancer.
\end{abstract}

\section{Introduction}

The high incidence of cancer leads to high mortality rates, with one in every four individuals being potential cancer patients (1). In general, most cancers are the result of various hereditary and environmental factors (2). Apoptosis, also known as programmed cell death, is a necessary genetically controlled process that is important in sustaining the balance

Correspondence to: Professor Ying-Li Pan, Department of Nursing, The Fourth Affiliated Hospital of China Medical University, East Road No. 4, Huanggu, Shenyang, Liaoning 110032, P.R. China

E-mail: cmu4p_pyl@163.com

Key words: apoptosis, caspases, polymorphism, cancer, meta-analysis of the development and maintenance of tissue homeostasis in multi-cellular organisms. Therefore, inappropriate regulation of apoptosis results in various human disorders, including cancer (3-6). The two main apoptotic pathways in humans, the extrinsic or death receptor-mediated pathway and the intrinsic or mitochondrial pathway, both utilize the caspase enzyme cascade. Caspases (cysteine and aspartic proteases, CAPS) are a cascade of enzymes that regulate apoptosis and are divided into initiator and executor caspases $(7,8)$. Caspase-2 belongs to initiator caspases and is primarily involved in the processing and activation of pro-inflammatory cytokines, and is involved in DNA damage-induced apoptosis. Caspase-1 and -5 have a similar structure and are predominantly involved in the maturation of pro-inflammatory cytokines $(1,8,9)$. Findings of previous studies demonstrated that caspase genes such as CASP-3, -7 and -8 , are associated with susceptibility to various types of human cancer (10-12). Investigations into the association between CASP-1, -2 and -5 and cancer risk are ongoing. Mittal et al (14) and Dong et al (13) have found that $\mathrm{C}$ allele carriers of rs507879 in CASP-5 were at higher risk of cancer. However, a contradictory result was found in another study where no significant association was identified between CASP-5 and cancer risk (15). Considering these inconsistent and inconclusive results, a Human Genome Epidemiology (HuGE) review and meta-analysis were conducted by including the most recent and relevant articles in order to identify statistical evidences to investigate the precise association between CASP-1, -2 and -5 and cancer risk.

\section{Materials and methods}

Literary search. Relevant papers published prior to October 1st, 2012 were identified through a search of Pubmed, Embase, Web of Science and CBM databases using the following terms: ('Genetic polymorphism' or 'polymorphism' or 'SNP' or 'gene mutation' or 'genetic variants') and ('neoplasms' or 'neoplasms' or 'cancer' or 'cancers' or 'carcinogenesis' or 'carcinoma') and ('caspase-1' or 'CASP-1' or 'caspase-2' or 'CASP-2' or 'caspase-5' or 'CASP-5'). The references from the eligible studies or textbooks were also reviewed manually to search for potentially eligible studies.

Inclusion and exclusion criteria. Inclusion criteria for the meta-analysis were: i) case-control or cohort study focused 

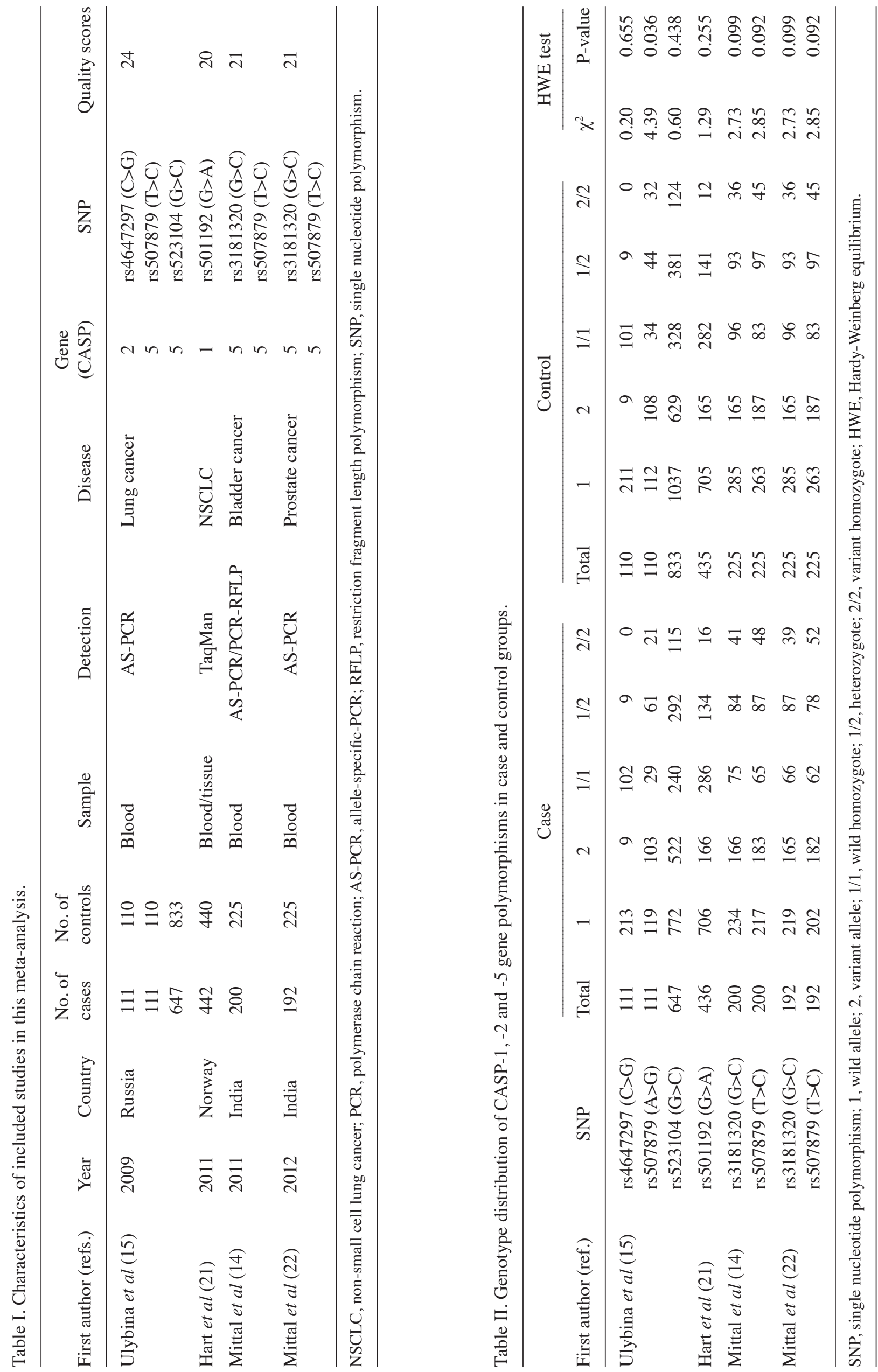
Table III. Meta-analysis of the association between polymorphisms of CASP-1, -2 and -5 genes and cancer susceptibility.

\begin{tabular}{|c|c|c|c|c|c|c|}
\hline Gene name & Gene/SNP & Case (n/N) & Control (n/N) & OR $(95 \% \mathrm{CI})$ & P-value & Effect model \\
\hline \multicolumn{7}{|l|}{ CASP-1 } \\
\hline \multirow[t]{2}{*}{ rs501192 (G>A) } & A allele & $166 / 872$ & $165 / 870$ & $1.00(0.79-1.28)$ & 0.97 & Fixed \\
\hline & A carrier & $150 / 436$ & $153 / 435$ & $0.97(0.73-1.28)$ & 0.81 & \\
\hline \multicolumn{7}{|l|}{ CASP-2 } \\
\hline \multirow[t]{2}{*}{ rs4647297 (C>G) } & $\mathrm{G}$ allele & $9 / 222$ & $9 / 220$ & $0.99(0.39-2.54)$ & 0.98 & Fixed \\
\hline & G carrier & $9 / 111$ & $9 / 110$ & $0.99(0.38-2.60)$ & 0.98 & \\
\hline \multicolumn{7}{|l|}{ CASP-5 } \\
\hline \multirow[t]{2}{*}{ rs507879 (T>C) } & $\mathrm{C}$ allele & $468 / 1006$ & $482 / 1120$ & $1.15(0.97-1.36)$ & 0.12 & Fixed \\
\hline & $\mathrm{C}$ carrier & $347 / 503$ & $360 / 560$ & $1.23(0.95-1.59)$ & 0.12 & \\
\hline \multirow[t]{2}{*}{ rs3181320 (G>C) } & $\mathrm{C}$ allele & $331 / 784$ & $330 / 900$ & $1.26(1.04-1.54)$ & 0.02 & Fixed \\
\hline & $\mathrm{C}$ carrier & $251 / 392$ & $258 / 450$ & $1.33(1.00-1.75)$ & 0.05 & \\
\hline \multirow[t]{2}{*}{ rs523104 (G>C) } & $\mathrm{C}$ allele & $522 / 1294$ & $629 / 1666$ & $1.11(0.96-1.29)$ & 0.15 & Fixed \\
\hline & $\mathrm{C}$ carrier & $407 / 647$ & $505 / 833$ & $1.10(0.89-1.36)$ & 0.37 & \\
\hline
\end{tabular}

SNP, single nucleotide polymorphism; OR, odds ratio; 95\% CI, 95\% confidence interval.

on the associations between CASP-1, -2 and -5 gene polymorphisms and cancer risk; ii) patients diagnosed with malignant tumors were required to be confirmed by pathological examinations; iii) published data concerning the frequency of alleles and genotypes was required to be sufficient; iv) studies were required to have been published in English or Chinese. Studies were excluded if they were: i) not a case-control or cohort study; ii) based on incomplete data; iii) duplicates of previous publications or iv) meta-analyses, letters, reviews or editorial articles.

Data extraction. Using a standardized form, data from published studies were extracted independently by two authors to populate the necessary information. For each study, the following characteristics were collected: the first author, year of publication, country, language, ethnicity, study design, number of subjects, source of cases and controls, pathological type, detecting sample, genotype method, allele and genotype frequencies and evidence of Hardy-Weinberg equilibrium (HWE) in controls. In case of conflicting evaluations, agreement was reached following discussion between the authors.

Quality assessment of included studies. Two authors independently assessed the quality of the studies according to modified STROBE quality score systems $(16,17)$. Forty assessment items associated with quality appraisal were used in this meta-analysis, with scores ranging from 0 to 40 . Scores of 0-20, 20-30 and 30-40 were defined as low, moderate and high quality, respectively. Disagreements were resolved through discussions between the authors.

Statistical analysis. The strength of the association between CASP-1, -2 and -5 gene polymorphisms and cancer susceptibility was measured by odds ratios (ORs) and $95 \%$ confidence intervals (CIs). The statistical significance of the pooled OR was examined using the $\mathrm{Z}$ test. Between-study variations and heterogeneities were estimated using Cochran's Q-statistic test with $\mathrm{P}<0.05$ indicating a statistically significant heterogeneity $(18,19)$. The effect of heterogeneity was quantified by using the $\mathrm{I}^{2}$ test (rang, 0-100\%), which represents the proportion of inter-study variability that can be contributed to heterogeneity instead of chance. When a significant $\mathrm{Q}$-test $(\mathrm{P}<0.05)$ or $\mathrm{I}^{2}>50 \%$ indicated that heterogeneity among studies existed, the random-effects model (DerSimonian and Laird method) was conducted for meta-analysis. Otherwise, the fixed-effects model (Mantel-Haenszel method) was used.

We also tested whether genotype frequencies of controls were in HWE using the $\chi^{2}$ test. Begg's funnel plots were used to detect publication biases. In addition, Egger's linear regression test, which measures funnel plot asymmetry using a natural logarithm scale of OR, was also used to evaluate the publication biases (20). To ensure the reliability and accuracy of the results, two reviewers assessed the data in the statistical software programs independently and obtained identical results. P-values were two-sided. Analyses were calculated using the Stata Version 12.0 software (Stata Corp., College Station, TX, USA).

\section{Results}

Characteristics of included studies. Four studies $(14,15,21,22)$ were included and 101 articles were excluded in the present meta-analysis. The flow chart of study selection is shown in Fig. 1. The publication year of involved studies ranged from 2009 to 2012 . In total, 1,592 cancer cases and 1,833 health controls were included in this meta-analysis. The patients diagnosed with cancer were also confirmed by pathological examinations. Cancer types included lung, bladder and prostate cancers. Five polymorphisms were evaluated, including rs501192 (G>A) in the CASP-1 gene, rs4647297 (C>G) in the CASP-2 gene, as well as rs507879 (T>C), rs3181320 (G>C) and rs523104 $(\mathrm{G}>\mathrm{C})$ in the CASP-5 gene. HWE test was conducted on the genotype distribution of the controls in the studies. Only the study by Ulybina et al (15) showed significant 


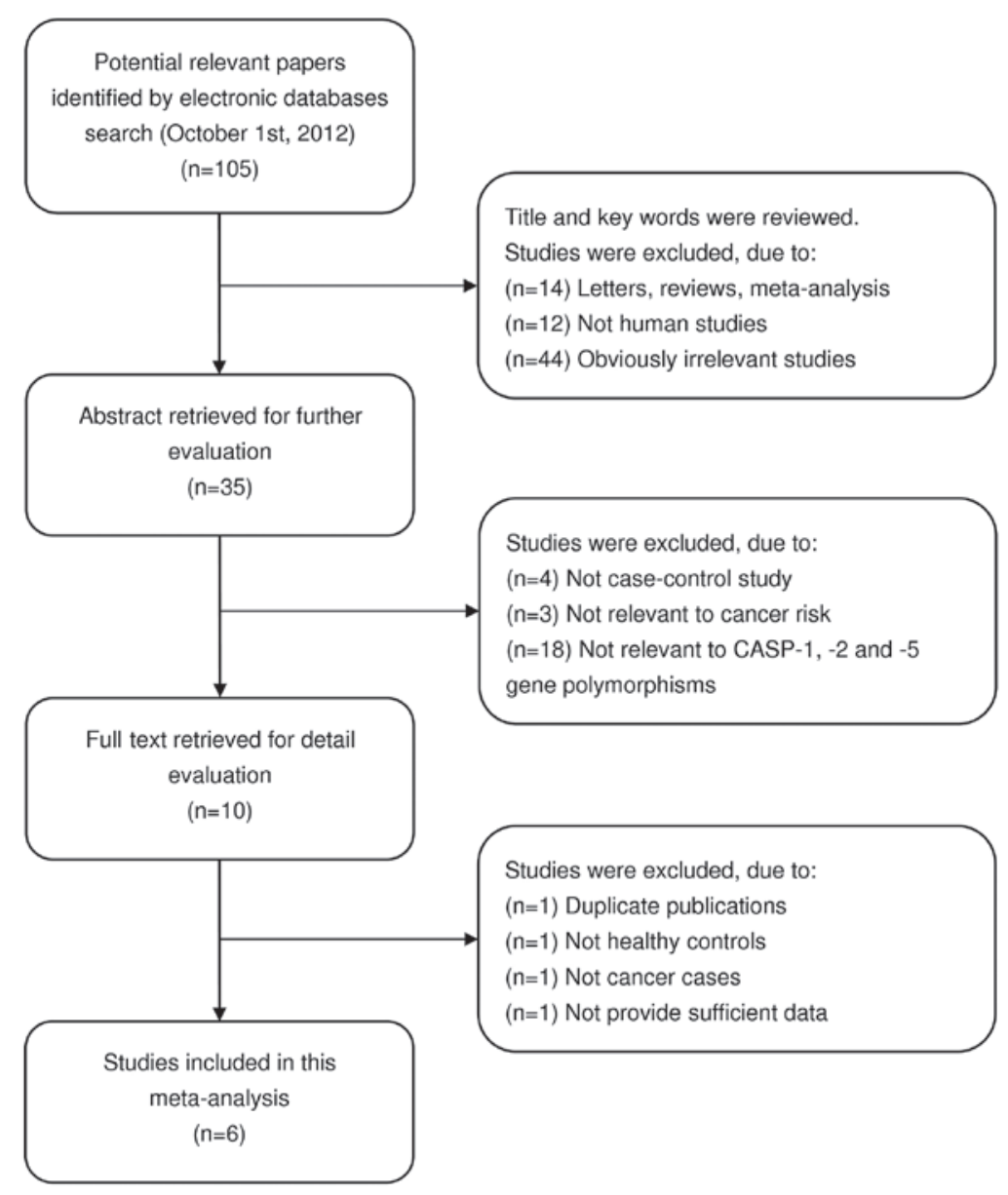

Figure 1. Flow chart shows study selection procedure.

deviation from HWE $(\mathrm{P}<0.05)$. The quality scores of included studies were $>20$ (moderate-high quality). The characteristics and methodological quality of the included studies are shown in Table I. The genotype distributions of CASP-1, -2 and -5 polymorphisms are presented in Table II.

Quantitative data synthesis. A summary of the meta-analysis findings of the associations between CASP-1, -2 and -5 gene polymorphisms and cancer risk is provided in Table III. Heterogeneity was not obvious in the polymorphisms (all $\mathrm{P}>0.05$ ), thus the fixed-effects model was used. When all the eligible studies were pooled into the meta-analysis, the results revealed that the $\mathrm{rs} 3181320 * \mathrm{C}$ allele/carrier in the CASP-5 gene were associated with increased risk of various cancers $(\mathrm{OR}=1.26 ; 95 \% \mathrm{CI}, 1.04-1.54 ; \mathrm{P}=0.020$ and $\mathrm{OR}=1.33$; $95 \% \mathrm{CI}$, 1.00-1.75; $\mathrm{P}=0.047$, respectively) (Fig. 2). Similar associations were not found in the rs501192, rs4647297, rs507879 and rs523104 polymorphisms (all $\mathrm{P}>0.05$ ).

Publication bias. Publication biases within the available research results may not be representative of the study results. Begg's funnel plot and Egger's linear regression test were performed to assess the publication biases of included studies. The shapes of the funnel plots did not reveal any evidence of obvious asymmetry of the CASP-1, -2 and -5 gene polymorphisms (Fig. 3). Egger's test also showed that there was no strong statistical evidence of publication bias $(\mathrm{t}=1.03, \mathrm{P}=0.343)$.

\section{Discussion}

Caspases, which play a key role in biochemical cell-suicide pathways, have been confirmed by a series of studies focusing on the association between caspase family members and cancer risk. Inappropriate regulation of apoptosis contributes to a number of human disorders (6). Caspases are also of fundamental importance in mediating apoptosis (11). Activation of caspases is essential in cell death commitment. Therefore, understanding of the mechanisms that underlie their activation is useful in clarifying the role caspases play in the induction of human disease (23). This is the most comprehensive meta-analysis examining CASP-1, -2 and -5 gene polymorphisms and their association with susceptibility to cancer. The strength of this study was based on the accumulation of published data, providing additional information to detect significant differences.

Caspase-1, the first identified caspase, was previously known as interleukin- $1 \beta$ processing enzyme (1). It may mediate cytokine production caused by tobacco smoke. The CASP-1 enzyme is essential for the cleavage of pro-IL1 protein into its active and mature forms and is involved in the formation and activation of inflammatory processes (24). Soung et al (25) have detected the probable role of inflammatory caspase in cancer. However, in the present meta-analysis, we investigated a polymorphism in CASP-1 gene rs501192 (G/A) and the results showed that neither the $\mathrm{A}$ allele nor the $\mathrm{A}$ allele 
A

\begin{tabular}{|c|c|c|}
\hline Study ID & OR $(95 \% \mathrm{CI})$ & Weight $\%$ \\
\hline Mittal et al-1 (2011) & $1.23(0.93,1.62)$ & 51.18 \\
\hline Mittal et al-2 (2011) & $1.30(0.98,1.72)$ & 48.82 \\
\hline Overall $\left(\mathrm{I}^{2}=0.0 \%, \mathrm{P}=0.764\right)$ & $1.26(1.04,1.54)$ & 100.00 \\
\hline 0.582 & .72 & \\
\hline
\end{tabular}

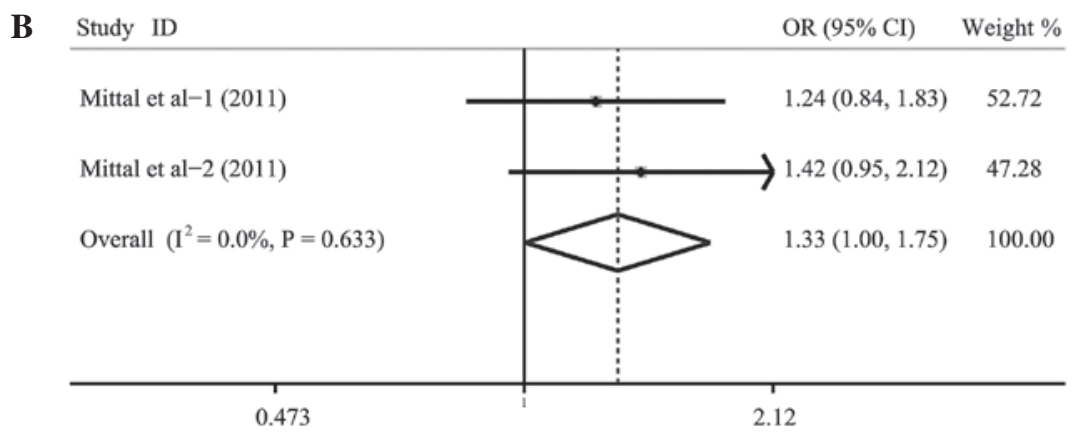

Figure 2. Forest plot shows the associations between rs3181320 polymorphism in the CASP-5 gene and cancer risk (A) C vs. G, (B) GC+CC vs. GG.

carrier of rs501192 exhibited any association with cancer risk. Caspase 2, encoded by the CASP-2 gene, cleaves other proteins belonging to the caspase family. Ulybina et al (15) have demonstrated that rs4647297 was not associated with cancer risk. Similarly, in the present study, no correlation was found between the $\mathrm{rs} 4647297 * \mathrm{G}$ allele/carrier in the CASP-2 gene and risk for various cancers. Caspase- 5 is also an enzyme that prototypically cleaves other proteins at an aspartic acid residue. Together with caspase 1, caspase 4, is important in the immune system. The CASP-5 mutations include nine mutations in exons, five mutations in introns and one in the 5'-untranslated region (1). To date, the biological function of the caspase-5 protein is poorly understood and is believed to play a role in various aspects of inflammation. In a recent study, a significant risk was observed in the $\mathrm{rs} 3181320 * \mathrm{C}$ carrier of the CASP-5 gene. In CASP-5 rs507879 T>C, C allele carriers were at a higher risk of breast cancer (14). By contrast, Ulybina et al (15) did not find any significant association with CASP-5 (15). In the present meta-analysis, we detected the polymorphisms of CASP-5, including rs507879 (T>C), rs3181320 (G>C) and rs523104 (G>C) and a significant association was identified with the $\mathrm{C}$ allele and $\mathrm{C}$ allele carrier of rs3181320 ( $\mathrm{G}>\mathrm{C})$. However, no association was detected in the $\mathrm{C}$ allele, $\mathrm{C}$ allele carrier of rs507879 (T>C) and rs523104 (G>C).

Similar to other meta-analyses, a number of limitations of this study should be addressed. Firstly, some relevant studies could not be included in our analysis due to incomplete raw data. Secondly, few relevant research articles were available and the sample size of this meta-analysis was limited. Thirdly, the source of heterogeneity among the studies could not be addressed. Fourthly, our meta-analysis was based on unad-

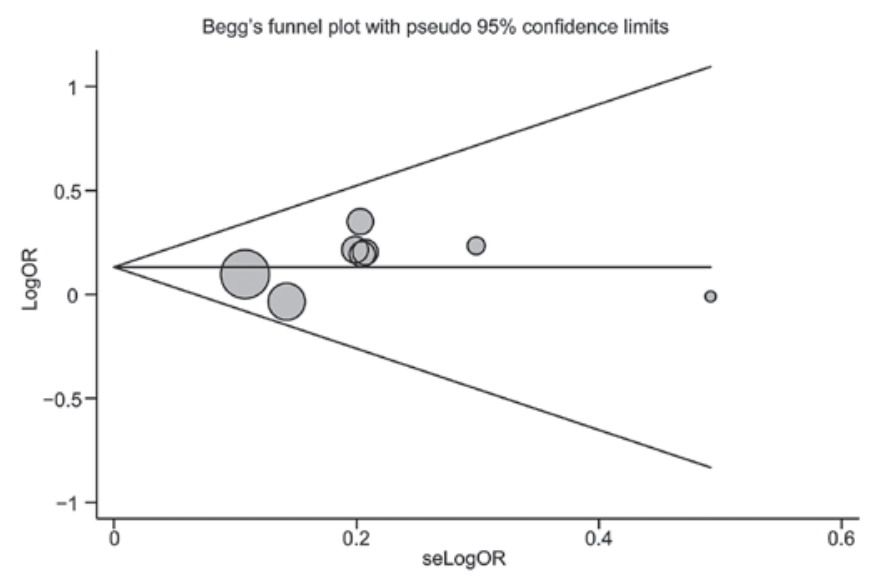

Figure 3. Begg's funnel plot of the meta-analysis of the associations between the CASP-1, -2 and -5 gene polymorphisms and cancer risk.

justed OR estimates as adjusted OR was not available in all published studies and when these studies were available, the OR was not adjusted using the same potential confounders, such as ethnicity, gender and geographic distribution. In addition, although the cases and controls of each study were well defined with similar inclusion criteria, potential factors that may have affected our results were not considered. Thus, additional investigation is required and our conclusions should be interpreted with caution.

In summary, this meta-analysis suggests that the rs $3181320 * \mathrm{C}$ allele/carrier in CASP-5 gene might be risk factors for cancer. However, few studies are currently available concerning this issue. Therefore, large-scale studies with 
an adequate methodological quality, and with appropriate controlling confounding factors should be conducted to obtain valid results.

\section{Acknowledgements}

We would like to acknowledge the helpful comments on this paper received from reviewers. We thank all our colleagues working in the Department of General Surgery, the Shenjing Hospital of China Medical University.

\section{References}

1. Ghavami S, Hashemi M, Ande SR, et al: Apoptosis and cancer: mutations within caspase genes. J Med Genet 46: 497-510, 2009

2. Dang TP: Notch, apoptosis and cancer. Adv Exp Med Biol 727: 199-209, 2012

3. Raff M: Cell suicide for beginners. Nature 396: 119-122, 1998.

4. Shivapurkar N, Reddy J, Chaudhary PM and Gazdar AF: Apoptosis and lung cancer: a review. J Cell Biochem 88: 885-898, 2003.

5. Hajra KM and Liu JR: Apoptosome dysfunction in human cancer. Apoptosis 9: 691-704, 2004.

6. Son JW, Kang HK, Chae MH, et al: Polymorphisms in the caspase- 8 gene and the risk of lung cancer. Cancer Genet Cytogenet 169: 121-127, 2006.

7. Degterev A, Boyce M and Yuan J: A decade of caspases. Oncogene 22: 8543-8567, 2003.

8. Kumar S and Dorstyn L: Analysing caspase activation and caspase activity in apoptotic cells. Methods Mol Biol 559: 3-17, 2009.

9. Siegel RM: Caspases at the crossroads of immune-cell life and death. Nat Rev Immunol 6: 308-317, 2006.

10. MacPherson G, Healey CS, Teare MD, et al: Association of a common variant of the CASP8 gene with reduced risk of breast cancer. J Natl Cancer Inst 96: 1866-1869, 2004.

11. Zhang X, Miao X, Sun T, et al: Functional polymorphisms in cell death pathway genes FAS and FASL contribute to risk of lung cancer. J Med Genet 42: 479-484, 2005.

12. Yang M, Sun T, Wang L, et al: Functional variants in cell death pathway genes and risk of pancreatic cancer. Clin Cancer Res 14: 3230-3236, 2008.
13. Dong LM, Brennan P, Karami S, et al: An analysis of growth, differentiation and apoptosis genes with risk of renal cancer. PloS One 4: e4895, 2009.

14. Mittal RD, Srivastava P, Mittal T, et al: Association of death receptor 4 , caspase 3 and 5 gene polymorphism with increased risk to bladder cancer in North Indians. Eur J Surg Oncol 37: 727-733, 2011.

15. Ulybina YM, Kuligina ESh, Mitiushkina NV, et al: Coding polymorphisms in Casp5, Casp8 and DR4 genes may play a role in predisposition to lung cancer. Cancer Lett 278: 183-191, 2009.

16. von Elm E, Altman DG, Egger M, et al: The Strengthening the Reporting of Observational Studies in Epidemiology (STROBE) statement: guidelines for reporting observational studies. Epidemiology 18: 800-804, 2007.

17. Zhang L, Liu JL, Zhang YJ and Wang H: Association between HLA-B*27 polymorphisms and ankylosing spondylitis in Han populations: a meta-analysis. Clin Exp Rheumatol 29: 285-292, 2011.

18. Higgins JP and Thompson SG: Quantifying heterogeneity in a meta-analysis. Stat Med 21: 1539-1558, 2002.

19. Zintzaras E and Ioannidis JP: Heterogeneity testing in meta-analysis of genome searches. Genet Epidemiol 28: 123-137, 2005.

20. Peters JL, Sutton AJ, Jones DR, et al: Comparison of two methods to detect publication bias in meta-analysis. JAMA 295: 676-680, 2006.

21. Hart K, Landvik NE, Lind H, et al: A combination of functional polymorphisms in the CASP8, MMP1, IL10 and SEPS1 genes affects risk of non-small cell lung cancer. Lung Cancer 71: 123-129, 2011.

22. Mittal RD, Mittal T, Singh AK and Mandal RK: Association of caspases with an increased prostate cancer risk in north Indian population. DNA Cell Biol 31: 67-73, 2012.

23. Kumar S: Measurement of caspase activity in cells undergoing apoptosis. Methods Mol Biol 282: 19-30, 2004.

24. Belogubova EV, Ulibina YM, Suvorova IK, et al: Combined CYP1A1/GSTM1 at-risk genotypes are overrepresented in squamous cell lung carcinoma patients but underrepresented in elderly tumor-free subjects. J Cancer Res Clin Oncol 132: 327-331, 2006.

25. Soung YH, Jeong EG, Ahn CH, et al: Mutational analysis of caspase 1,4 , and 5 genes in common human cancers. Hum Pathol 39: 895-900, 2008. 